\title{
Approche et traitement des cybercrises
}

\section{Dealing with cybercrises}

Oléagineux, Corps Gras, Lipides. Volume 7, Numéro 5, 441-2, Septembre - Octobre 2000, Dossier : Sécurité sanitaire des aliments et industrie

Auteur(s) : Elodie BOURDEAU, Alain PAJOT, Startem, 60, rue de Ponthieu, 75008 Paris.

Résumé : Avec l'explosion d'Internet, de nombreuses entreprises du secteur agro-alimentaire sont aujourd'hui amenées à faire face à des crises d'un genre nouveau qui voient le jour sur Internet, et qu'on appelle cybercrises. Pour les y aider, la société Startem, créée en 1994 et regroupant une soixantaine de consultants, propose aux entreprises de ce secteur des moyens afin de gérer au mieux ces crises. Ainsi, parmi d'autres activités, Startem recueille et analyse tout ce qui se dit ou s'écrit dans les médias sur chacun de ses clients, et ce dans 95 pays et 23 langues. De plus, Startem propose d'accompagner ses clients dans la gestion d'une crise en aidant, par exemple, à la mise en place de sites Internet/Intranet de crise.

Mots-clés : communication, crise, information, international, Internet, médias.

Summary: Some food sector companies are given a bad press via Internet. For example, wrong information can produce catastrophic and sometimes irreversible consequences. In order to anticipate or deal with new kind of crisis, the Startem company offers to observe and process information into 95 countries and 23 languages. Startem also gives some advice to manage these increasing cybercrisis.

Keywords: communication, crisis, information, international, Internet, the media.

\section{ARTICLE}

Si Internet représente aujourd'hui un formidable outil d'information et de communication, il s'avère, également dans certains cas, être source de vulnérabilité pour les entreprises. En effet, nombre d'entre elles ont déjà fait l'objet de campagnes, pour la plupart particulièrement virulentes, via le réseau Internet. II est aisé, pour un internaute, de distiller des informations pouvant nuire à l'image d'une entreprise ou d'un produit alimentaire, et ce d'autant plus que le cadre réglementaire est imparfait.

Ces campagnes peuvent prendre différentes formes : il peut s'agir de la diffusion de canulars, d'attaques informatiques, mais aussi de campagnes de désinformation lancées par les concurrents. Ainsi, des bruits diffusés, via Internet, sur la mise en faillite de Belvédère, fabricant de bouteilles 
d'alcool, ont entraîné une perte d'environ 600 millions de francs de sa capitalisation boursière. Un autre exemple concerne les bananes du Costa-Rica, qualifiées sans aucune preuve de " bananes tueuses ». Plus récemment, certains médias affirmaient que les paysans indiens, ruinés par Monsanto, vendaient leur rein pour acheter leurs semences.

Ces informations sont d'autant plus dangereuses que, vraies ou fausses, elles ont tôt fait d'être répandues aux quatre coins de la planète, et ce sans aucune médiation. II est parfois de bon ton de critiquer les journalistes et les médias mais, sur Internet, on regrette l'absence de régulateurs de l'information.

De tous les secteurs d'activité concernés par ces cybercrises, le secteur agro-alimentaire, aussi bien les grands groupes que les PMI et les PME, et les filières agricoles se révèlent aujourd'hui particulièrement exposés. La veille sanitaire, le traitement de l'information, la détection et la maîtrise des crises à travers le réseau Internet (les cybercrises) sont devenus des formes d'action et de réponse que les entreprises ne peuvent plus ignorer.

Créée en 1994, la société Startem s'est spécialisée dans ce domaine. Regroupant une soixantaine de consultants, Startem recueille, traite, analyse et diffuse l'information médiatisée nationale et internationale (voir son site : http://startem.net).

Dans le cadre de veille sanitaire, Startem recueille et analyse tout ce qui se dit ou s'écrit sur chacun de ses clients, dans 95 pays et dans 23 langues, soit 75000 publications ainsi traitées (25 000 titres électroniques et 50000 sur papier).

Cette veille permanente se révèle un moyen efficace d'anticipation des crises : elle permet d'être informé en temps réel dès la diffusion dans les médias d'une information sur l'entreprise, ses marchés ou ses concurrents.

Par ailleurs, l'analyse approfondie de la presse permet aux sociétés de déterminer, par exemple, les thèmes suscitant l'intérêt des journalistes, ou encore la distorsion entre " l'image perçue " et « l'image désirée » de l'entreprise ou de ses produits.

Startem, qui consacre près de $40 \%$ de ses activités au secteur agro-alimentaire, a ainsi développé un véritable observatoire dans ce secteur, comme par exemple lors des crises OGM (figures 1 et 2).

Pendant les premiers jours de la crise qui a touché Coca-Cola en juin 1999, suite à plusieurs cas d'intoxication signalés en Europe, une veille réalisée pour Le Figaro s'est traduite par l'identification de plus de 20000 messages hostiles circulant déjà sur le Net, alors que l'entreprise fermait ses installations en Belgique.

Dans ce contexte, la société propose également d'accompagner ses clients dans la gestion des crises, en définissant des moyens de réponses sous forme de sites Internet/Intranet de crise.

Ainsi, en juin dernier, lorsque des traces d'organismes génétiquement modifiés ont été trouvées dans des semences de maïs importées et du soja. Face à l'ampleur des protestations, Dominique Voynet, ministre de l'Environnement a, dans un premier temps, envisagé de demander l'arrachage de tous les plants incriminés, alors que le maïs transgénique est autorisé en France. Avec l'autorisation de son client, I'Association générale des producteurs de maïs (AGPM), Startem peut mentionner qu'elle a 
alimenté un site Internet, créé une cellule de concertation et formé les agriculteurs en région à répondre aux questions des journalistes de radio et de télévision.

Il est indispensable d'avoir conscience de ce que représente Internet aujourd'hui, aussi bien du point de vue de la " veille sanitaire " que des réponses à y apporter dans le cadre de la gestion de crise. Or cette prise de conscience n'est à l'heure actuelle qu'émergente.

Illustrations

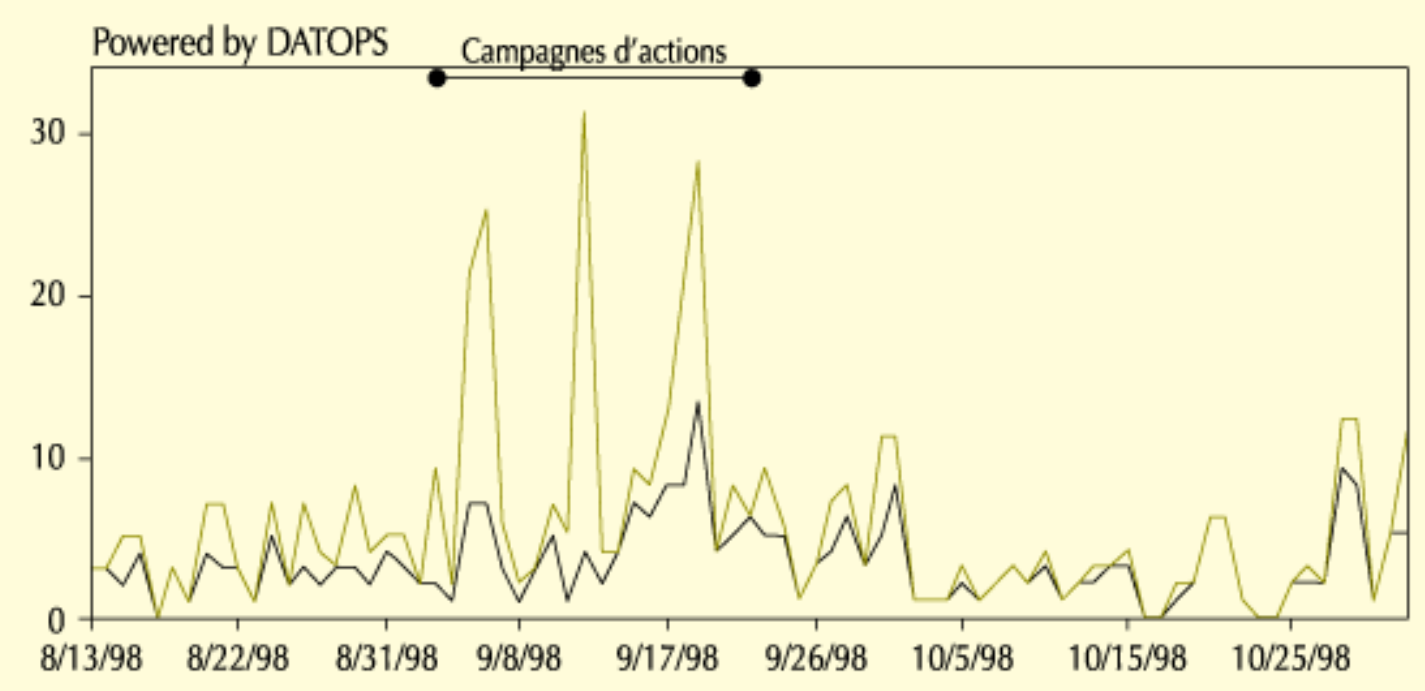

Figure 1. Crises OGM. Volume d'informations et auteurs sur News Group. Source :

Atlantic Intelligence. 


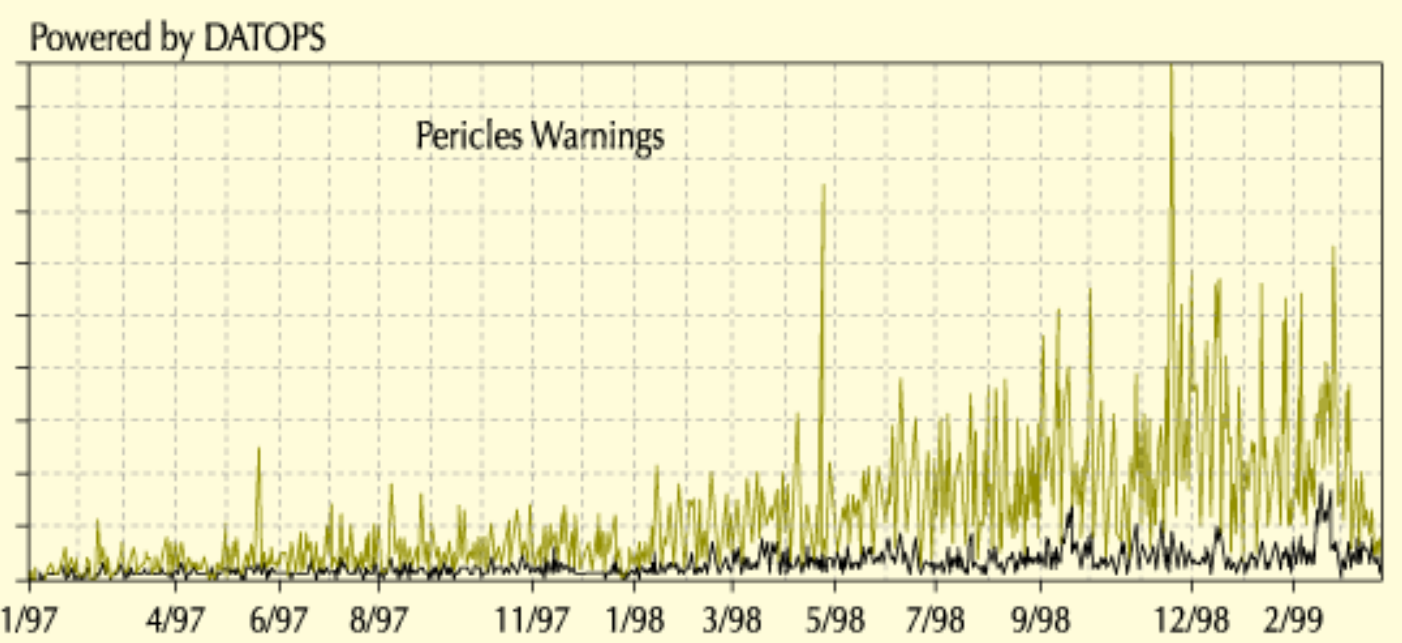

Figure 2. Débat sur les OGM. Volume d'informations dans le web visible. Source : Atlantic Intelligence. 